\title{
NIR Spectrometer used for Material Modeling with Neural Networks
}

\author{
Nigel Yee \\ Electrotechnology Department, \\ Unitec Institute of Technology, \\ Private Bag 92025, Victoria Street West, \\ Auckland 1142, New Zealand
}

\begin{abstract}
Near infrared multi-spectral image analysis is a tool used for non-destructive determination of biological material properties. In this investigation a custom built imaging spectrometer is constructed and used for the image spectra instrumentation and tests are performed on this instrument to determine its spectral resolution and spectral range; a biological data set (moisture in potato crisps) is then captured using this instrument and this data set is modeled using near infrared multi-spectral image analysis. A common problem with near infrared multi-spectral quantitative image measurements is light scatter and light non-linearity resulting from sample shape contours/curvatures and optical aberrations from optical component selection/layout. In this paper we detail an imaging spectrometer and the use of orthogonal signal correction preprocessing combined with a neural network full spectrum model for measurement of material property.
\end{abstract}

Keywords-Neural network, near infrared spectrometer, biological material

\section{Introduction}

Chemicals present in biological material absorb electromagnetic radiation at certain wavelengths and thus electromagnetic radiation supplied to the objects under investigation will be absorbed by the sample as opposed to being reflected back from the sample into the spectral instrumentation. Measurement of the amount of electromagnetic radiation reflected off the biological material and the application of spectral calibration modeling techniques allows a determination of the biological materials composition to be made. Near infrared (NIR) imaging spectroscopy is considered to be the compilation and analysis of electromagnetic radiation between the wavelengths of 700$2500 \mathrm{~nm}$ at different spatial locations. NIR spectroscopy is a popular non-destructive measurement tool because it is low cost and robust $[1,2,3]$.

A major problem with near infrared spectral data is lighting non-linearity and light scatter. Orthogonal Signal Correction (OSC) is a tool used for pre-processing spectral data $[4,5,6]$ which corrects for instrumental drift, bias and scatter effects in electromagnetic spectral analysis and has proved popular within the NIR region of the spectrum $[7,8,9]$. OSC functions by separating the systematic variation into orthogonal factors, where the factors contain the variation within the $\mathbf{X}$ matrix data that is not correlated with the $\mathbf{y}$ vector data. This implementation of OSC is combined with a neural network full spectrum model to make a prediction of moisture content in the biological sample.

This article details a purpose built low cost NIR instrumentation and a demonstration of using OSC with the neural network calibration model to remove lighting irregularities from the spectra and determine biological material properties. The task is the determination of the moisture content in potato crisp near infrared spectra.

\section{METHOD}

\section{A. Apparatus}

The aim of this work is to accurately measure biological material properties (potato crisp moisture content) using low cost near infrared imaging spectroscopy instrumentation. To achieve this, a low cost near infrared spectral imaging instrument was built according to a tested design [10]. This instrumentation used a low cost silicon Charged Coupled Detector (CCD) that operated from $400 \mathrm{~nm}$ to $1100 \mathrm{~nm}$ and obtained image spectra.

A picture of the image spectrometer components mounted on an optical layout table is shown in Fig. 1. At the entrance to the optical system a narrow slit images a slice of the sample and then a collimating lens collimates rays from the image slice of the object onto the diffraction grating. The rays reflected off the diffraction grating (which now contain spectral information) are focused (by the camera lens) onto the CCD (see Table 1 for component list). Spatial information in the vertical direction is captured by the optics as columns on the detector and each row of the CCD measures spectral information, corresponding to one vertical position at the slit 
Table 1. Spectrometer components.

\begin{tabular}{|l|l|}
\hline Component type & Component selected \\
\hline Imaging lens & Olympus $50 \mathrm{~mm}$ \\
\hline Entrance slit & $18 \times 120 \mu \mathrm{m}$ \\
\hline Order Blocking filter & Glass filter 665nm cut on \\
\hline Efficiency adjustment & Glass filter 385 peak \\
\hline Collimating lens & $100 \mathrm{~mm}$ Cooke triplet \\
\hline Diffraction grating & 300 lines mm ${ }^{-1}$ \\
\hline Focusing lens & Olympus 35mm instruments TC245 \\
\hline CCD detector & $\begin{array}{l}\text { Texas } \\
755 \times 244 \\
6.4 m m x 4.8 m m ~\end{array}$ \\
\hline
\end{tabular}

(see Fig. 2). For reflectance measurements, the object is illuminated from the front with a diffuse halogen lamp and in practice the instrument is mounted above a conveyor (see Fig. 3 ), which acts as a translation stage and allows scanning of the objects to build spectral images.

\section{B. Spectral Dataset Collection}

A total of 120 potato crisp samples were presented to the spectrometer (see Fig. 3) to generate a data set of 120 spectral images. The measurements were taken in reflectance mode and the mathematical treatment of the spectral variables was $\log _{10}(1 / R)$ where $\mathrm{R}$ represents the reflectance value at a specified wavelength.

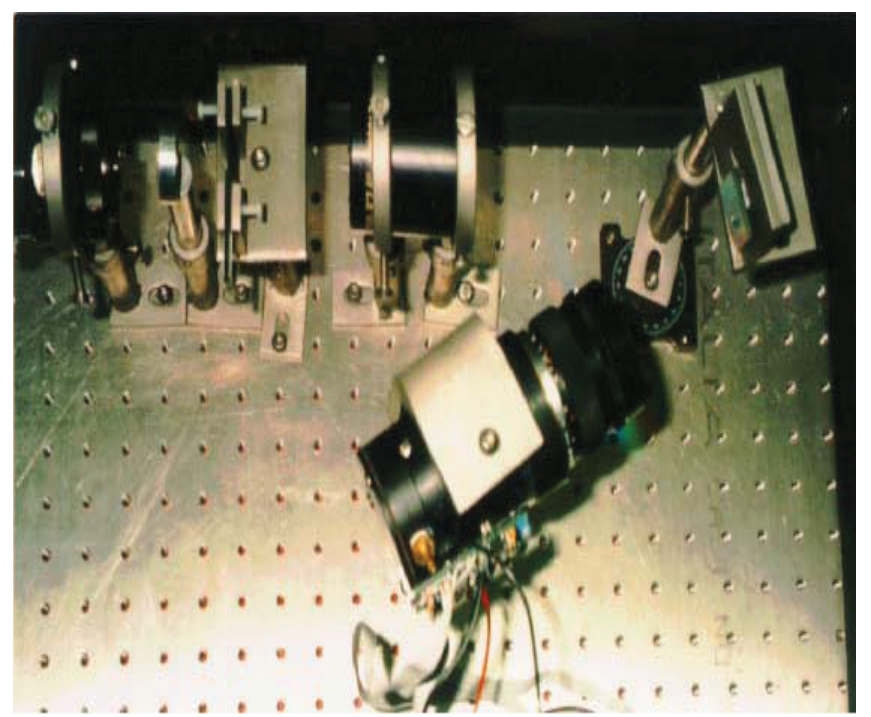

Figure 1. Components of image spectrometer mounted on an optical layout table.

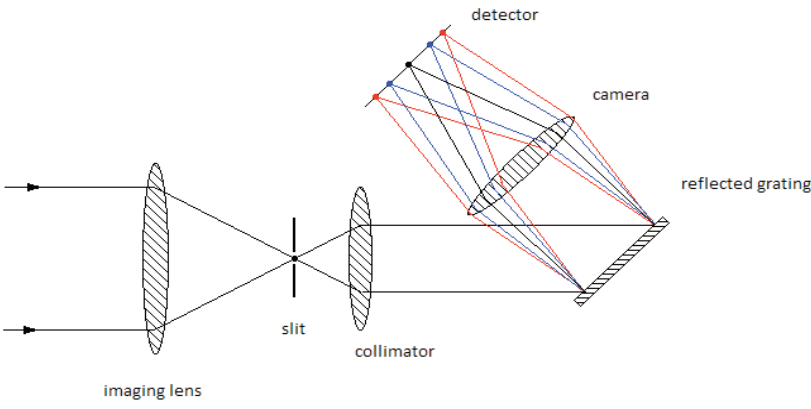

Fig 2. Layout of long slit image spectrometer components.

The potato crisp samples had the moisture content varied using equilibration [11] to achieve a $4-35 \%(0.04-0.35$ relative moisture) variation in moisture content. The moisture content was measured after the spectra was collected using the drying method [12].

\section{Orthogonal Signal Correction}

The OSC algorithm forms factors that contain variation in $\mathbf{X}$ not correlated with $\mathbf{y}$ and functions by using a subtraction from the $\mathbf{X}$ matrix, of scores $\mathbf{t}$ and loadings $\mathbf{p}$, that contain the largest amounts of variability in $\mathbf{X}$, uncorrelated to $\mathbf{y}$. The OSC problem is posed as $\mathbf{t}=\mathbf{X r}$, where

$$
\operatorname{Max}\left(\mathbf{t}^{\mathrm{T}} \mathbf{t}\right)
$$

subject to constraints $\mathbf{t}^{\mathrm{T}} \mathbf{y}=0$ and $\mathbf{r}^{\mathrm{T}} \mathbf{r}=1$.

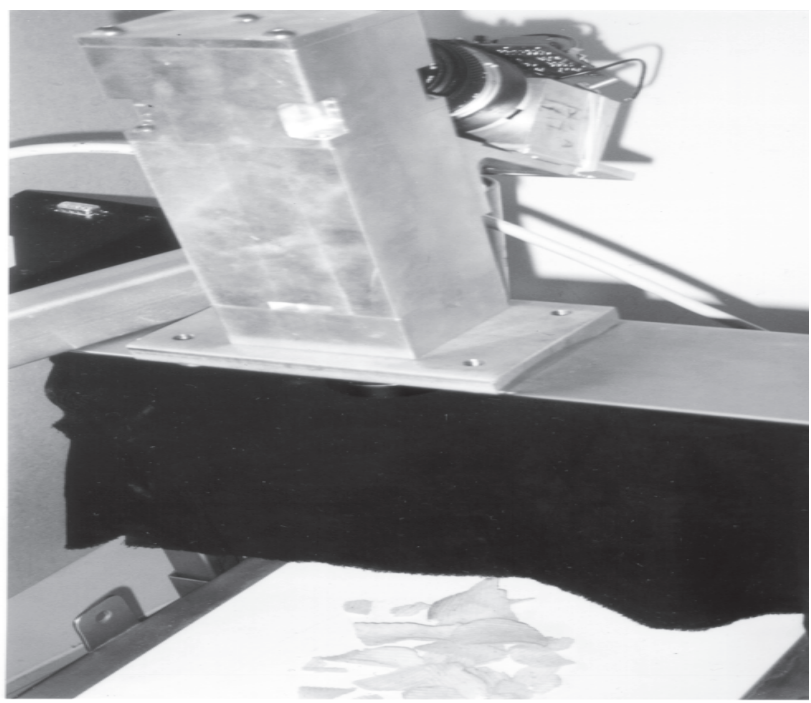

Figure 3. Spectrometer mounted on a conveyor with sample product. 
Steps 1-4 are now used to produce OSC factors.

For step 1, we use

$$
\mathbf{M}=\mathbf{I}-\mathbf{X}^{\mathrm{T}} \mathbf{y}\left(\mathbf{y}^{\mathrm{T}} \mathbf{X} \mathbf{X}^{\mathrm{T}} \mathbf{y}\right)^{-1} \mathbf{y}^{\mathrm{T}} \mathbf{X}
$$

for step 2, the weighting matrix $\mathbf{X}$ is formed

$$
\mathbf{Z}=\mathbf{X} \mathbf{M} \text {. }
$$

Eigenvalues $\lambda$ and eigenvectors $\mathbf{c}$ of $\mathbf{Z}^{\mathrm{T}} \mathbf{Z}$ are computed to produce scores $\mathbf{t}$ and loadings $\mathbf{p}$ in steps $3-4$.

Step 3, scores are calculated by

$$
\mathbf{t}_{\mathrm{i}}=\lambda^{-1 / 2} \mathbf{M} \mathbf{X}^{\mathrm{T}} \mathbf{c}_{\mathrm{i}}
$$

and step 4, loadings are calculated by

$$
\mathbf{p}_{\mathrm{i}}=\mathbf{X}^{\mathrm{T}} \mathbf{t}_{\mathrm{i}} / \mathbf{t}_{\mathrm{i}}^{\mathrm{T}} \mathbf{t}_{\mathrm{i}},
$$

Additional scores and loading vectors are calculated using the corrected $\mathbf{X}_{\mathrm{i}}$ matrix and re-inserting the corrected matrix into steps $1-4$

$$
\mathbf{X}_{\mathrm{i}}=\mathbf{X}-\mathbf{t}_{\mathrm{i}} \mathbf{p}_{\mathrm{i}}^{\mathrm{T}}
$$

\section{Prediction using OSC}

The loadings and weightings from the steps 1-4 are stored and used in the prediction matrix where $\mathbf{T}=\left[\mathbf{t}_{1}, \mathbf{t}_{2}, \ldots \mathbf{t}_{\mathrm{n}}\right]$ is a matrix containing all the $\mathbf{t}$ vectors and $\mathbf{P}=\left[\mathbf{p}_{1}, \mathbf{p}_{2}, \ldots \mathbf{p}_{\mathrm{n}}\right]$ is a matrix containing the $\mathbf{p}$ load vectors used.

$$
\mathbf{X}_{\text {new }}=\mathbf{X}-\mathbf{T} \mathbf{P}_{\text {new }}{ }^{\mathrm{T}} \text {, }
$$

\section{E. Neural Network Correction}

The neural network used for the function approximation problem was a back propagation neural network utilising a Jacobian matrix (see Fig 4). This neural network consisted of three layers: an input layer, an intermediate hidden layer and an output layer and is configured in the feed forward direction [13] with one input node per spectral wavelength on the input layer which provided 755 input nodes in total. A number of trials were performed using different numbers of nodes within the hidden layer for the purpose identifying a structure that would give an acceptable preliminary result in a reasonable time without undue stress being placed on the computing infrastructure. From these trials a structure containing one hidden layer that comprised of 6 nodes and an output layer comprising of one node was selected for training and was used for the purpose of reporting the neural networks performance in trial experiments. The output layer was trained with the moisture content data and the performance of the network was determined using dot product correlation coefficient which in this case provides an indication of the strength of the correlation between spectral information and moisture content in the samples.

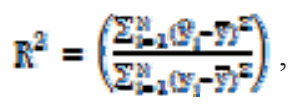

where $y_{i}$ is the measured value of the moisture for the $i^{\text {th }}$ object $\square$ of the mean of the moisture, $\hat{y}_{i}$ is the moisture value computed by the neural network [14].

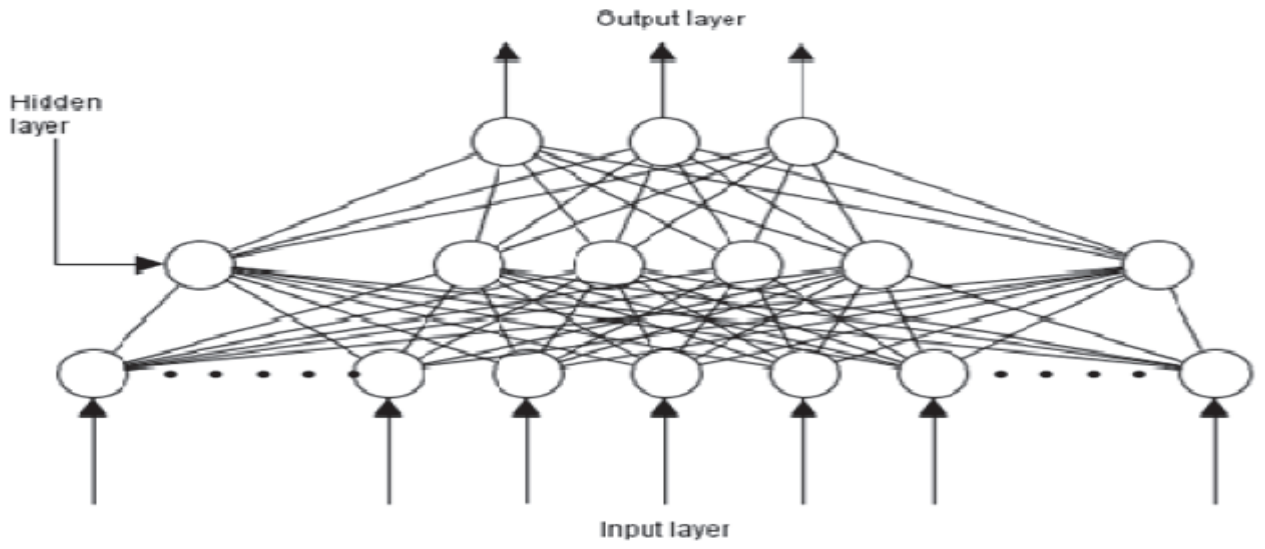

Fig 4. Neural network with input layer, hidden layer and output layer. 


\section{F. Data Analysis}

A Pentium work station running two $2.4 \mathrm{GHz}$ processors and the Windows 7 operating system was used to capture the data. The spectrometer camera used a parallel port which was interfaced with the work station. Image acquisition drivers were written in Visual $\mathrm{C}++$ so that data from the spectrometer could be captured and displayed in Matlab [15]. The data analysis involving neural network modeling and orthogonal correction analysis was undertaken using Matlab R13 on this machine. The optical modeling was undertaken using Zemax OpticStudio [16] on this machine.

\section{RESULTS AND DISCUSSION}

\section{A. Spectrometer Calibration Results}

A mercury-argon lamp was used to examine the spectral range and the spectral resolution of the spectrometer. An image acquired from an argon lamp is shown in Fig. 5, this image shows the vertical direction containing the spatial information while the horizontal direction corresponds to spectral information. From this image it is clear that there are prominent spectral peaks which appear as much lighter vertical lines. There appear to be more spectral peaks on the left hand side of the image between column number 0-250 (horizontal axis) which corresponds to the lower wavelengths. Fig. 6 shows a plot of spectral intensity vs. wavelength that was obtained from data corresponding to the spatial position 72 (vertical axis of Fig. 5). This plot shows the sharp peaks

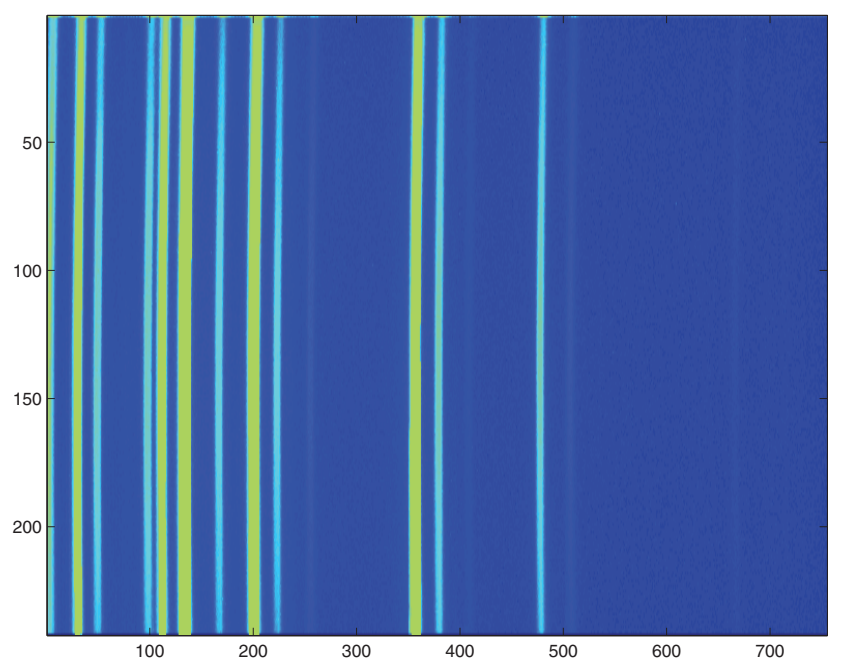

Figure 5. Image captured by the spectrometer of an argon lamp (spatial position on vertical axis and spectral information on horizontal axis). characteristic of argon gas. The theoretical spectral resolution of this instrument is $3.3 \mathrm{~nm}$ but the practical resolution is determined by the optical components used and their resulting optical aberrations. Inspection of two prominent spectral peaks located at $794.8 \mathrm{~nm}$ and $800.6 \mathrm{~nm}$ indicates a spectral resolution of $5.8 \mathrm{~nm}$ is obtainable in practice.

\section{B. Full Spectrum OSC Model Results}

Fig. 7, shows a multi-spatial multi-spectral image of a sample potato crisp. The potato crisp was located between positions 0 and 125 in the spatial dimension (vertical axis). It is clearly observed that lighting non-linearity exists in the spatial dimension for a fixed spectral point. A data set was obtained by capturing images of common potato crisp spectra located between spatial co-ordinates 0-125 (spectra was extracted from these images at spatial position 72 to form the data set). The data was randomly split with 100 of the spectra in the calibration set, and 20 of the spectra in the prediction set.

The calibration data set was pre-processed with one OSC factor and the spectral data was then run through the neural network and the predicted relative moisture content vs. the measured relative moisture content was plotted (see Fig. 8). The correlation coefficient for this plot is $\mathrm{R}^{2}=0.6489$. This correlation coefficient is considered an acceptable result and suggests that a reasonable model can be formed to predict moisture content in potato chips using near infrared imaging.

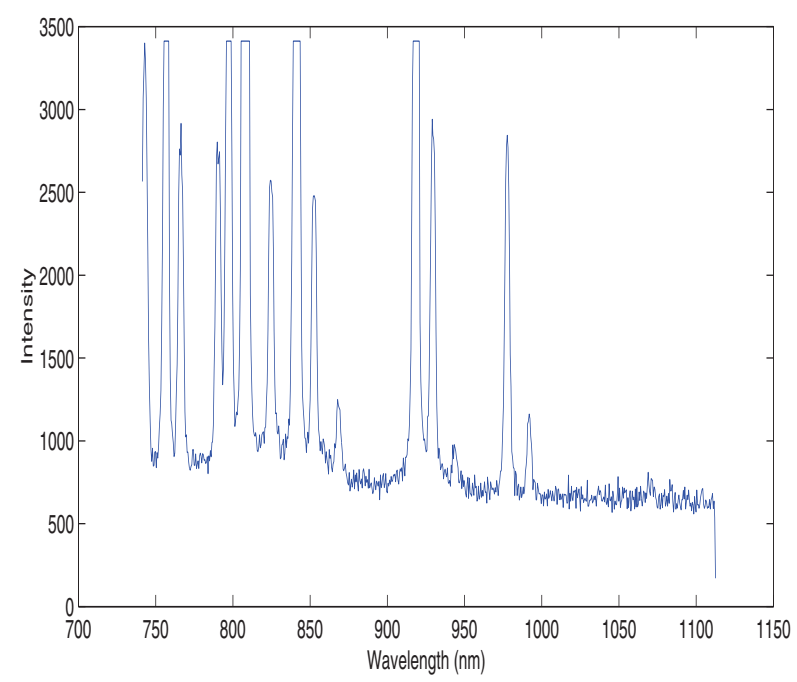

Figure 6. Plot of spectra from an argon lamp vs. wavelength. 


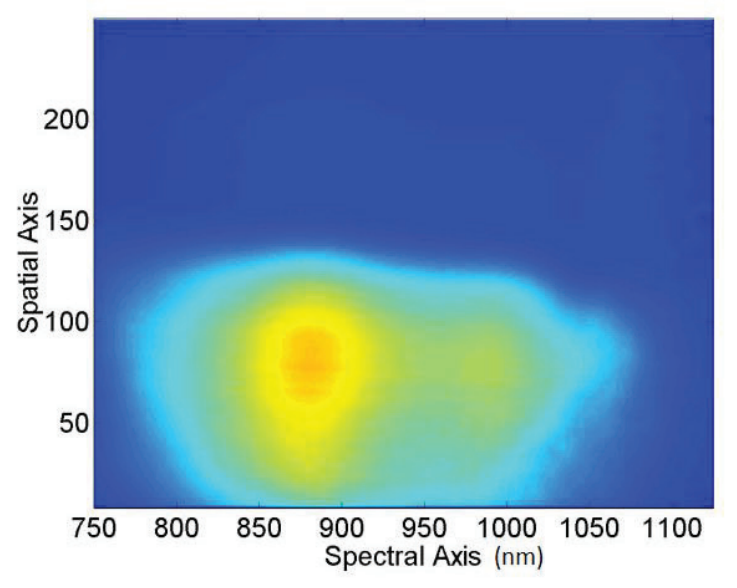

Figure 7. A spectral image captured for a potato crisp using an imaging spectrometer.

\section{Future work}

The system described in this paper uses a silicon based detector and silicon detectors have an upper spectral limit of $1100 \mathrm{~nm}$, as moisture has a two fundamental absorption bands in the infrared region $(\mathrm{O}-\mathrm{H}$ symmetric stretching at $2734 \mathrm{~nm}$ and $\mathrm{O}-\mathrm{H}$ asymmetric stretching at $2662 \mathrm{~nm}$ ) the only moisture absorption bands observable by this instrument are overtones of a fundamental band. These overtones lack the intensity of the fundamental absorption bands and tends to produce more convoluted data signals and thus a more powerful spectrometer with greater spectral range will provide an improved result. Presently, we have acquired a Zeiss NIR Spectrometer that uses an InGaAs detector and has a range from $900 \mathrm{~nm}$ to $1700 \mathrm{~nm}$ and we intend to use this instrument for future investigations.

During this investigation a major problem has been the irregular geometry of the samples causing light to scatter and this has never been adequately dealt with using scatter correction pre-processing methods. In quantitative NIR spectral analysis the imaging spectrometer can measure across multiple spatial points (the equivalent of 244 single point spectrometers in this example) however the single spatial point spectrometer with a fiber optic probe input tends to provide stronger signals. A limitation of the approach that uses an imaging spectrometer is that the input to the imaging spectrometer is not readily coupled to fiber optic probes and for this reason we intend to use a single spatial point spectrometer with the fiber optic probe input for our future investigations.

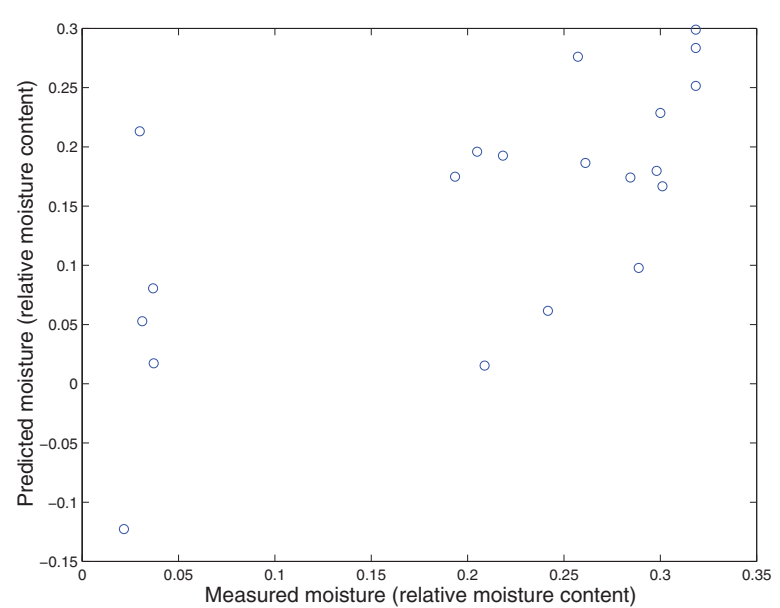

Figure 8. Plot of predicted moisture content vs. measured moisture content for the prediction set.

\section{REFERENCES}

[1] K.B. Walsh, J.A. Guthrie and J.W. Burney: "Application of commercially available low-cost, miniature NIR spectrometers to the assessment of sugar content of intact fruit" Australian Journal of Plant Physiology. vol. 27(12), pp. 1175, 2000,

[2] S. D. Osborne and R. Künnemeyer: "A low-cost system for the grading of kiwifruit" Journal of Near Infrared Spectroscopy. vol. 7, pp 9, 1999.

[3] F. Chauchard, R. Cogdill, S. Roussel, J.M. Roger and V. Bellon-Maurel, "Application of LS-SVM to non-linear phenomena in NIR spectroscopy: development of a robust and portable sensor for acidity prediction in grapes," Chemometrics and Intelligent Laboratory Systems, vol.71(2), p 141, 2004.

[4] T. Fearn, "On orthongonal signal correction", Chemometrics and intelligent laboratory systems, vol 50, pp47, 2000.

[5] R. Balabin and S Smirnov, "Melamine detection by mid- and nearinfrared (NIR/MIR) spectroscopy: a quick and sensitive method for dairy products analysis including liquid milk", Talanta, vol 85, pp 562, 2011.

[6] H. Gu, Z. Pan, B. Xxi, V. Asiago, B. Musselman and D. Rattery, "Principal component directed partial least squares analysis for combining nuclear magnetic resonance and mass spectrometry data in metabolomics: Application to the detection of breast cancer", Analytica chimica acta, vol 686, 2011.

[7] S. Wold, H. Antti, F. Lindgren and J. Ohman, "Orthogonal signal correction of near-infrared spectra", Chemometrics and Intelligent Laboratory Systems, vol. 44, pp 175, 1998.

[8] R. Balabin, E. Lomakina and R. Safieva, "Neural network (ANN) approach to biodiesel analysis: Analysis of biodiesel density, kinematic viscosity, methanol and water contents using near infrared (NIR) spectroscopy”, Fuel, vol 90, pp 2007, 2011. 
[9] J. Boulet and J. Roger, "Pretreatments by means of orthogonal projectsions", Chemometrics and intelligent laboratory systems, vol 117 , pp 61, 2012.

[10] P. Martinsen: Quantitative near infrared imaging spectroscopy of fruit. PhD thesis, University of Auckland, Auckland, New Zealand, 1999.

[11] Moisture Systems Corporation. December Applications Newsletter. Moisture Systems Corporation, 117 South Street, Hopkinton, MA, USA. 1993.

[12] L. Nollet, Handbook of food analysis. Marcel Dekker, New York, 1996.

[13] H. Rumelhart, G.E. Hinton and R.J. Williams. "Learning internal representations by error propagation,” Nature. vol. 323, pp 533, 1986 ,
[14] D. Burns and E. Ciurczak, Handbook of Near-Infrared Analysis, $3^{\text {rd }}$ ed, CRC Press, Boca Raton, Florida, pp142, 2001.

[15] N. R. Draper and H. Smith, Applied regression analysis, $2^{\text {nd }}$ ed., Wiley, New York, 1981.

[16] MATLAB 13.0 and Statistics Toolbox, The MathWorks, Inc., Natick, Massachusetts, United States.

[17] OpticStudio13.0 Zemax, LLC, Redmond, Washington, United states. 